\title{
Videojuegos y mundos de ficción. De Super Mario a Portal.
}

CP 2014 , Vol.4-Nº, pp. 114-117. ISSN 2014-6752. Girona (Catalunya) PUIG BORRÀS, Núria: Videojuegos y mundos de ficción. De Super Mario a Portal. Recibido: 21 /07/2014 - Aceptado: 24/07/2014

Videojuegos y mundos de ficción. De Super Mario a Portal.

Antonio J. Planells

Editorial: Ediciones Cátedra

Colección: Signo e Imagen

Año: 2015 - Madrid

Número de páginas: 250

ISBN: 978-84-376-3349-7

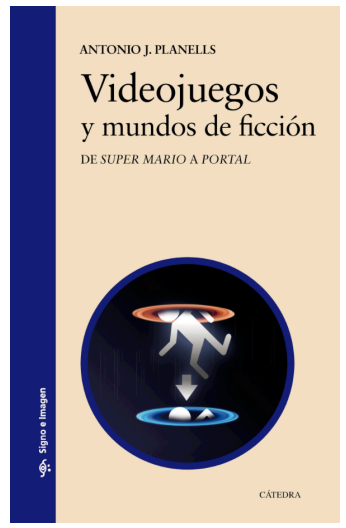

\section{Núria PUIG BORRÀS}

Universitat de Girona. nuria.puig@udg.edu

Videojuegos y mundos de ficción de Antonio J. Planells es una investigación teórica que desemboca en el análisis del entramado de los mundos de ficción de ocho videojuegos. El autor propone un sistema teórico-práctico de análisis construido a partir de aportaciones de la teoría de la ficción y la teoría de los mundos posibles. La propuesta se denomina "teoría de los mundos ludoficcionales" y está especialmente diseñada para comprender los videojuegos como sistemas lúdicos que participan en un entorno ficcional de de manera distinta a como lo hacen medios tradicionales como el cine o la televisión.

El libro está estructurado en tres partes: una primera parte dedicada a la teoría de los mundos posibles y a los mundos de ficción; una segunda parte dedicada a los mundos de ficción, los espacios jugables y los videojuegos, y una tercera parte dedicada a los análisis de casos y al planteamiento de los mundos ludoficcionales en los géneros del videojuego.
Los primeros capítulos están dedicados a la clarificación terminológica de conceptos que provienen de diferentes áreas de conocimiento de las humanidades y de las ciencias sociales. Al mismo tiempo, el autor adapta la terminología y los métodos de análisis a las particularidades de los videojuegos. A lo largo de los primeros capítulos el acercamiento al objeto de estudio se produce desde la filosofía analítica, la filosofía del lenguaje, la narratología y las distintas aportaciones en el campo de la literatura y los estudios fílmicos. En esta parte del libro destacan las referencias a Platón, Aristóteles, Russel, Lewis o Leibniz.

Los capítulos centrales se dedican a la noción de narrativa planteando el debate entre ludología y narratología y otras posiciones intermedias, de entre las que cabe destacar las ideas y la muestra de videojuegos con potencialidad narrativa analizada por Henry 
Jenkins. En este apartado, el autor plantea la noción de mundo ludoficcional. Planells nos acerca a los Game Studies y a los autores y textos fundamentales de esta línea de estudio. A lo largo de todo el libro hay que destacar la revisión de teorías y autores relacionados con el tema que ofrece al lector una visión muy amplia y completa de teorías y autores de ámbitos diversos. Es de interés la referencia a los estudios sobre videojuegos, los Game Studies. En relación a los Game Studies podemos leer:

“A finales de los años noventa Espen Aarseth publicó Cybertext: perspectives on ergodic literature (1997), una de las obras más influyentes para la textualidad digital. En ella, el autor nórdico propone una lectura especial de los juegos y los videojuegos como cibertextos, alejándolos de las nociones tradicionales del hegemónico hipertexto (Bolter, 1991; Landow 1995). El cibertexto es una perspectiva que se utiliza ante textos dinámicos, es decir, aquellos que poseen el carácter ergódico por el cual se genera, en sentido amplio, una cadena de eventos mediante el cual se genera, en sentido amplio, una cadena de eventos mediante los esfuerzos no triviales (nontrivial efforts) de uno o más usuarios (Aarseth, 1997, pág 94)." (Planells, 2015: 75)

Planells destaca el papel del usuario en la perspectiva propuesta para el cibertexto:

"Mientras que en el paradigma hipertextual el papel del usuario es exploratorio o selectivo ante una estructura predeterminada, el cibertexto dota al interactor de capacidad configurativa en tanto que le permite generar nuevos textos partiendo de un sistema subyacente (págs. 58-65)" (Planells, 2015: 75)

En el capítulo 4, se definen los conceptos de gameplay, game design y play. Y se describen las característica fundamentales de los mundos ludificcionales. En primer lugar, los mundos ludificcionales están estructurados en sistemas de mundos posibles cohesionados, estables y autónomos. En segundo lugar, son modelos estáticos y diseñados para ser autentificables por uno o más jugadores. En tercer lugar, establecen mecanismos de retroalimentación y dependencia ente su dimensión lúdica y su dimensión de ficción. En cuarto lugar, los mundos ludoficcionales están metalépticamente naturalizados. $\mathrm{Y}$, en quinto lugar, participan del modelo ecológico del transmedia.

"En relación con otros medios, el tránsito ficcional ha sido muy fructífero tanto en un sentido como en otro. Gracias al establecimiento de un potente star system -Mario Bros, Sonic, Lara Croft, Kratos, Link o Solid Snake, entre otros- y unas longevas sagas con ricos mundos de ficción -Mass Effect (Bioware, 2008-2012) o Resident Evil (Capcom, 1996-2012)-, los videojuegos han generado libros, cómics, juegos de mesa y, por supuesto, películas. Y es justamente en el medio cinematográfica donde el ideal transmedia parece fracasar de manera evidente con unas obras que desvirtúan totalmente el marco ficcional del juego. (Planells, 2015: 110)

El punto de vista, el espacio y la temporalidad -entre otros aspectoscompletan este capítulo que acaba con un ejemplo de videojuego: Dragon Age. Un juego que no propone una estructura lineal sino un mundo ludificcional abierto con un conjunto de mundos posibles primarios que hay que alcanzar y unos mundos posibles secundarios que proponen digresiones $y$ aventuras paralelas. (Planells, 2015: 148)

El exhaustivo marco teórico de las dos 
primeras partes nos conducen al análisis de algunos videojuegos. En la tercera parte, el autor analiza ocho juegos: Portal 2, Alice Madness Returns, Resonance, Alan Wake, Starcraft II: Wings of Liberty, The Elder Scrolls V: Skyrim, Diablo III y The Movie.

El primer juego analizado es Portal 2 (Valve Corporation, 2011) y tiene lugar en un laboratorio subterráneo abandonado. El entramado de mundos de Portal 2 manifiesta una linealidad absoluta al enlazar cada mundo posible primario de manera consecutiva. (Planells, 2015: 176). El autor destaca en relación a este juego la lucha de poder y el choque entre los sentimientos del ser humano y el pensamiento lógico de la máquina. La mecánica de juego se basa en un sistema de portales en el que cada mundo posible se identifica con una habitación cerrada.

Alice Madness Returns (Spicy Horse, 2011) parte de la obra literaria Las aventuras de Alicia en el país de las maravillas (1865). Los dos videojuegos dirigidos por el diseñador American McGee parten del canon, la obra literaria, para construir un nuevo universo mucho más oscuro, demente y violento. (Planells, 2015: 182). Este juego transcurre de forma lineal con la particularidad que lo real y lo imaginado se entrelaza. Los mundos de enlazan en dos niveles de la ficción, que obedecen, en sentido estricto, al submundo real efectivo y al submundo imaginado-temido de Alicia. (Planells, 2015: 183)

El juego Resonance (XII Games, 2012) muestra cuatro retratos de los protagonistas principales que se corresponden con cuatro mundos posibles primarios. El objetivo de estos primeros mundos es el de presentar a los personajes e ir tejiendo, poco a poco, los lazos que acabarán relacionándolos entre sí. Este tipo de presentación de particularidades ficcionales se ve claramente influido por obras corales de la narrativa modular como 21gramos (Alejandro González Iñárritu, 2003) o Magnolia (Paul Thomas Anderson, 1999), en las que el seguimiento de cada personaje es el camino para entender la relación entre cada uno de ellos. (Planells, 2015: 189)

En el caso de Alan Wake (Remedy Entertainment, 2010), según Planells a nivel macroestructural la propuesta de Remedy Entertainment se asemeja más a las series de televisión que a los videojuegos, con una estructura episódica cuyos episodios abren y cierran mundos posibles narrativos. Destaca una estructura lineal y cinematográfica.

Con Starcraft II: Wings of Liberty (Blizzard Entertainment, 2010) se enmarca en el grupo de los juegos de estrategia en tiempo real. Starcraft II se construirá desde dos niveles que, en su relación, tejerán un rico mundo ludificccional. Por un lado, la aparición de las constelaciones particulares y la combinatoria de submundos proporcionan una explicación ficcional a la dimensión estratégica y, por otro lado, la resolución de cada uno de los conflictos se encauza mediante las convenciones y las experiencias de juego propias del género. (Planells, 2015: 203)

The Elder Scrolls V: Skyrim (Bethesda Game Studios, 2011) es un videojuego de rol que despliega grandes mundos de juego muy ricos en particularidades ficcionales, ciudades y elementos naturales, así como misiones épicas y combates heroicos contra seres de todo tipo. Junto a esta exaltación de la ficción, el rol permito la construcción progresiva del personaje jugador, desde su raza, profesión y estética hasta sus habilidades, poderes y, en ocasiones, marcos éticos e ideológicos.(Planells, 2015: 208)

Diablo III (Blizzard Entertainment, 2012) es un juego de rol en línea y multijugador. $L a$ disposición macroestructural de Diablo III se establece en cuatro actos en dos niveles. (Planells, 2015: 215) Segun Planells, Diablo III expone un mundo multipersonal y cooperativo que asigna las relaciones dialécticas y constructivas a la zona segura (...) y las relaciones físicas y destructivasconstructivas en el nivel del juego de mazmorras.(Planells, 2015: 216)

Finalmente, The Movies (Lionhead Studios, 2005) supone la evolución de los 
simuladores sociales y el fenómeno de Los Sims. La dimensión macroestructural estática en The Movies se concentra en un espacio de juego que constituye el único mundo posible primario. Junto a este, y en el mismo contexto diegético, se establece un mundo posible secundario que permite la creación de distintas películas. (Planells, 2015: 220)

Con estos ocho juegos analizados en la última parte del libro vemos un abanico de ejemplos de distintas tipologías de juego, diferentes estructuras ficcionales y distintas formas de crear mundos de ficción en los videojuegos. Con este análisis observamos también la aplicación de la teoría de los mundos ludoficcionales para conocer mejor las estructuras de los videojuegos y su relación con otras formas de narración como son el cine o la televisión.

En referencia a las fuentes utilizadas en el estudio, destacar la gran cantidad de ejemplos que están recogidos en una Ludografia completa que incluye juegos desde principios de los años 90 hasta la actualidad. La lista de juegos aporta también datos del estudio desarrollador. Un interesante inventario de ejemplos que enriquecen las páginas de este estudio académico y que juntamente con la revisión teórica ofrece un útil recorrido por teorías y autores.

La obra va dirigida a un público principalmente académico interesado en aspectos relacionados con la narrativa, el relato, el análisis del discurso y la filosofía. Videojuegos y mundos de ficción constituye un paso más en el camino científico y de análisis de los videojuegos con una propuesta de análisis de videojuegos desde las aportaciones de la teoría de la ficción y la teoría de los mundos posibles que responde por igual a la perspectiva lúdica del juego y a la perspectiva ficcional del mundo que evoca (Planells, 2015).

\section{Forma de citación}

PUIG BORRÀS, Núria: Videojuegos y mundos de ficción. De Super Mario a Portal. Revista Communication Papers, $N^{\circ} 6$, páginas 114 a 117. Departamento de Filología y Comunicación de la Universidad de Girona. Recuperado el de de 2 de: http://www.communicationpapers.es 